Laser Chem., 1997, Vol. 17, pp. 161-174

Reprints available directly from the publisher Photocopying permitted by license only
(C) 1997 OPA (Overseas Publishers Association)

Amsterdam B.V. Published under license under the Harwood Academic Publishers imprint, part of The Gordon and Breach Publishing Group.

\title{
NON-LINEAR SIMULTANEOUS TWO-PHOTON EXCITATION ENERGY TRANSFER IN THE WRONG DIRECTION
}

\author{
M. NICKOLEIT, A. UHL and J. BENDIG* \\ Institute of Organic and Bioorganic Chemistry, Humboldt University, \\ Hessische Str. 1-2, D-10115 Berlin, Germany
}

(Received 23 October 1996)

The simultaneous two-photon excitation energy transfer (SEET) was demonstrated for the first time using trichromophoric model compounds. Two identical donors $(A-$ antenna) were covalently linked to an energy acceptor unit ( $T$-target) with different energy levels preventing energy transfer of a single photon. At high intensity illumination (laser exposure) of a trichromophoric system $A \sim T \sim A$ ( $A$-fluorescein, erythrosin; $T-E$ stilbene), sufficient to excite both of the appended donor subunits, population of the target excited state may occur via simultaneous energy transfer of two photons, one from each donor. In order to restrict reverse energy transfer from the higher energy target to the lower energy donor(s) it is necessary that the excited target unit undergoes an efficient photoreaction. In the investigated case this was achieved by photoisomerization of the stilbene unit used for monitoring of the SEET.

Keywords: Two-photon process; energy transfer; photoisomerization; stilbene

\section{INTRODUCTION}

The phenomenon of radiationless electronic excitation energy transfer (EET)

$$
D^{*}+A \stackrel{k_{\mathrm{EET}}}{\longrightarrow} D+A^{*}
$$

has been intensively studied both theoretically and experimentally [1, 2]. The precondition for EET from $D^{*}$ (electronically excited donor

\footnotetext{
${ }^{*}$ Corresponding author.
} 
molecule) to $A$ (acceptor molecule) is a partial overlap of the donor fluorescence band $F^{D}(\tilde{v})$ and the acceptor absorption band $\varepsilon^{A}(\tilde{v})$. The EET process can be the result of long range Coulombic (dipol-dipol, Förster mechanism) and short range electron-exchange interaction.

According to Förster [3], the rate coefficient for Coulomb excitation energy transfer $k_{\mathrm{EET}}(C)$ is described by

$$
k_{\mathrm{EET}}(C)=\frac{9000(\operatorname{In} 10) \kappa^{2} \varphi_{f, o}^{D}}{128 \pi^{5} n^{4} N_{A V} \tau_{f, o}^{D} r^{6}} J
$$

where $\kappa^{2}$ is the orientation factor [2], $\varphi_{f, 0}^{D}$ is the donor fluorescence quantum yield in the absence of the acceptor, $n$ is the refractive index, $N_{A V}$ is Avogadro's number, $r$ is the distance between donor and acceptor and $J$ is the overlap integral defined by

$$
J=\int_{0}^{\infty} F^{D}(\tilde{v}) \varepsilon^{A}(\tilde{v}) \tilde{v}^{-4} d \tilde{v} .
$$

According to Dexter [4], the rate coefficient for electron-exchange excitation energy transfer $k_{\mathrm{EET}}(E)$ depends on the overlap of the emission spectrum and the absorption spectrum of $A$, too,

$$
k_{\mathrm{EET}}(E)=\frac{2 \pi}{\hbar} Z^{2} J
$$

where the parameter $Z$ is obviously related to the electronic matrix element for electron-exchange energy transfer $\left(Z^{2} \propto \exp (-2 r / l), l\right.$ is the van der Waals radius of the donor-acceptor pair).

The simultaneous two-photon excitation energy transfer (SEET) from two excited energy donors $A^{*}$ ( $A$-antenna unit) to one energy acceptor ( $T$-target unit)

$$
A^{*} \sim T \sim A^{*} \stackrel{k_{\text {SEET }}}{\longrightarrow} A \sim T^{*} \sim A
$$

is still unknown. In this paper we discuss the concept of SEET and investigate the deactivation behavior of the covalently linked $A \sim T \sim A$ system 1 and 3 containing fluorescein or erythrosin (antenna unit) and $E$-stilbene (target unit) (Tab. I). The SEET enables a deep UV photochemistry at high intensity long wavelength excitation. 


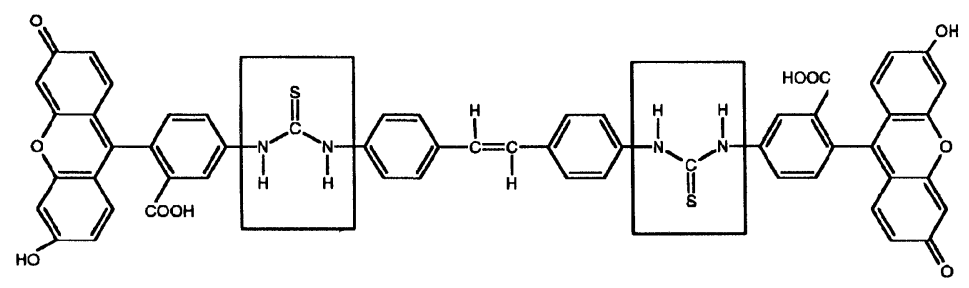

TABLE I Investigated compounds

\begin{tabular}{|c|c|c|}
\hline No. name & antenna & target \\
\hline $\begin{array}{ll}1 & E-4,4^{\prime} \text {-Di(fluorescein-5"'-yl-thioureanyl)stilbene } \\
2 & E-4-\text { Fluorescein-5"-yl-thioureanyl-stilbene } \\
3 & E-4,4^{\prime} \text {-Di(erythrosin-5" } \\
4 & E-4-\text {-Eryl-thioureanyl)stilbene } \\
& \end{array}$ & $\begin{array}{l}\text { fluorescein } \\
\text { fluorescein } \\
\text { erythrosin } \\
\text { erythrosin }\end{array}$ & $\begin{array}{l}E \text {-stilbene } \\
E \text {-stilbene } \\
E \text {-stilbene } \\
E \text {-stilbene }\end{array}$ \\
\hline
\end{tabular}

\section{CONCEPT OF SEET}

The principle of SEET (eq. 4) is shown in Figure 1. The energy of the excited state of the target unit is marked higher than the excited state energy of the antenna system. The requirements of a successful proof of the SEET are:

1. Provided that the SEET has taken place, the rate coefficient of the "common" energy transfer (eqs. 1, 3, 5)

$$
A \sim T^{*} \sim A \stackrel{k_{\mathrm{EET}}}{\longrightarrow} A \sim T \sim A^{*}
$$

will be very high. Therefore, the proof of the existence of $T^{*}$ requires an adjustment of the spectroscopic properties of $A$ and $T$ resulting in a small overlap integral (eq. 2) and a small deactivation rate coefficient of $T^{*}$ by EET (eq. 5).

2. Resulting from the strong competition of the EET (eq. 5) and the photophysical and/or photochemical deactivation of $T^{*}$ it is necessary to use target units characterized by a very high rate coefficient of the fluorescence of $T^{*}$ or by a rapid chemical deactivation of $T^{*}$. In that case the chemical reaction is the indication of a previously occured SEET. 

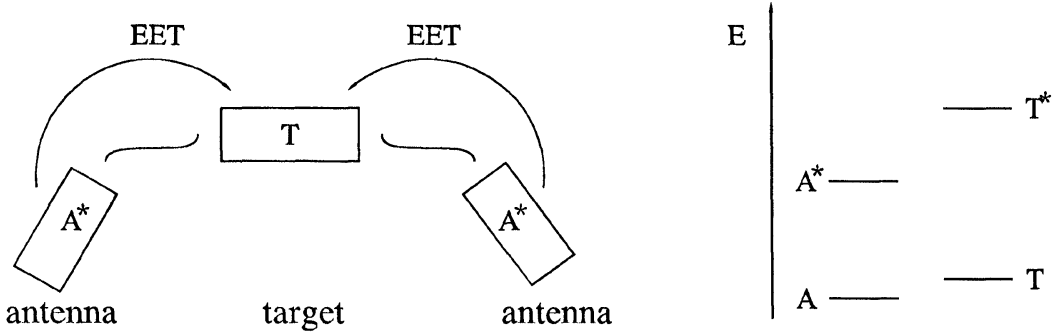

$$
\text { precondition: } \mathrm{E}\left(\mathrm{A}^{*}\right)<<\mathrm{E}\left(\mathrm{T}^{*}\right) ; 2 \mathrm{E}\left(\mathrm{A}^{*}\right) \geq \mathrm{E}\left(\mathrm{T}^{*}\right)
$$

FIGURE 1 Scheme of the simultaneous excitation energy transfer (SEET).

The model compound $E-4,4^{\prime}$-di(fluorescein-5"'-y1-thioureanyl)-stilbene 1 (nomenclature according to Haugland and Larison [5]) was used to varify this concept. The fluorescein chromophore is the antenna unit, the $E$-stilbene represents the target unit. The successful SEET will be shown by the $E, Z$-isomerization of the stilbene at (high intensity) fluorescein excitation (eq. 6).

$$
A^{*} \sim T_{E} \sim A^{*} \stackrel{k_{\mathrm{SEET}}}{\longrightarrow} A \sim T_{E}^{*} \sim A \stackrel{k_{\text {iso }}}{\longrightarrow} A \sim T_{Z} \sim A
$$

To prove the concept of SEET the corresponding dichromophoric systems $A \sim T 2$ and 4 (Tab. I) were also investigated.

\section{EXPERIMENTAL}

\section{Materials and Preparations}

All preparations were carried out under yellow light.

1: $0.2 \mathrm{~g}\left(7.07 \cdot 10^{-4} \mathrm{Mol}\right)$ of $4,4^{\prime}$-diaminostilbene dihydrochloride (Aldrich) were dissolved in $10 \mathrm{ml}$ ethanol with an additional catalytic amount of triethylamine. $0.54 \mathrm{~g}\left(1.41 \cdot 10^{-3} \mathrm{Mol}\right)$ of fluorescein-5'-yl-isothiocyanate (Aldrich) dissolved in $20 \mathrm{ml}$ ethanol were added. The obtained red crystals of 1 were isolated after standing over night. 1 was purified by recrystallization from ethanol; m.p.: $>350^{\circ} \mathrm{C}$. Yield of chromatographic pure material: $98 \%$. 
2: Synthesis of 2 was carried out analogously to 1 starting from $0.2 \mathrm{~g}$ $\left(1.02 \cdot 10^{-3} \mathrm{Mol}\right)$ of 4 -aminostilbene, prepared according to Harder et al. [9], and $0.39 \mathrm{~g}\left(1.02 \cdot 10^{-3} \mathrm{Mol}\right)$ fluorescein-5'-ylisothiocyanate. The reaction solution was evaporated to dryness and the residue was recrystallized from ethanol; m.p.: $>350^{\circ} \mathrm{C}$. Yield of chromatographic pure material: $95 \%$.

3/4: Synthesis of 3 and $\mathbf{4}$ were done according to $\mathbf{2}$ using erythrosin- $5^{\prime}-$ yl-isothiocyanate (Aldrich) in equimolaric amounts.

\section{Instrumentation and Measurements}

The registration of the UV/VIS absorption spectra was realized with a U-3410 spectrophotometer (Hitachi) combined with a computer. The emission spectra were recorded using a fluorescence spectrophotometer MPF-2A (Hitachi-Perkin-Elmer). The solutions were thermostated.

All investigations were done using aqueous/ethanolic solutions (10:90). For the reason of the dependence of the excited state properties of the dyes upon the $\mathrm{pH}$ value (band position, life time and quantum yield of the fluorescence, Figs. 2, 3), all solutions were alkalized with sodium hydroxide to $\mathrm{pH}=8$.

The absolute fluorescence quantum yields $\varphi_{f}$ were determined at 298 $\mathrm{K}$ according to the relative method [6] using fluorescein as a standard $\left(\varphi_{f}=0.90\right.$ [7] $)$. The determination of the fluorescence decay times is described [8].

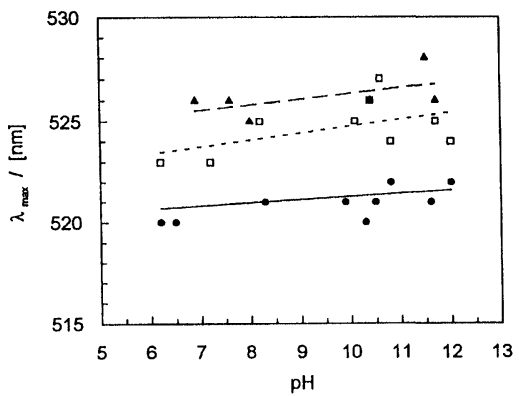

FIGURE 2 Fluorescence band maxima at different pH-value; $\bullet$ : fluorescein sodium, $\square: \mathrm{N}$-phenyl-N'-(fluorescein-5"-yl)-thiourea, $\boldsymbol{\Delta}: \mathbf{1}$. 


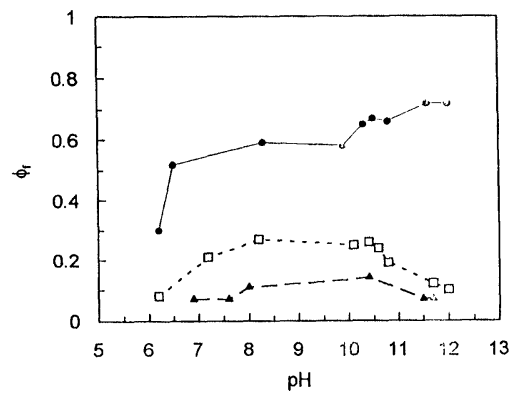

FIGURE 3 Fluorescence quantum yields at different $\mathrm{pH}$-value; $\bullet, \square$ and $\boldsymbol{\Delta}$ see Figure 2.

HPLC was carried out using the following conditions: HPLC pumps 422/422S with HPLC mixer M 494 (Kontron Instruments); mobile phase methanol-water ( $95: 5$ for $\mathbf{1}$ and 3; $60: 40$ for $\mathbf{2}$ and 4); flow rate $0.5 \mathrm{ml} \mathrm{min}-1$; Model 7125 Syringe Loading Sample Injector (Rheodyne Inc.); Knauer column $(250 \mathrm{~mm} \times 4 \mathrm{~mm})$ filled with LiChrosorb RP $18(5 \mu \mathrm{m})$; DAD 440 (Kontron Instruments); Data System DS 450-MT2 for data evaluation.

The low intensity photolysis was realized using a high pressure mercury lamp HBO 500 (Oriel) with controlled light intensity and a metal interference filter. The laser exposure experiments were carried out with an $\mathrm{Ar}^{+}$-Laser (Coherent, Inc., $\lambda_{\mathrm{exc}}=488 \mathrm{~nm}$ for 1 and 2 and $\lambda_{\text {exc }}=514 \mathrm{~nm}$ for 3 and 4) and/or a dye laser (FL 80, ZWG, $\lambda_{\mathrm{exc}}=500 \mathrm{~nm}$ for 1 and $2, \lambda_{\mathrm{exc}}=514 \mathrm{~nm}$ for 3 and 4 using Coumarin 307, Lambdachrome) pumped by an $\mathrm{XeCl}$ excimer laser (EMG 103, Lambda Physik).

\section{RESULTS}

\section{Spectroscopic Properties}

The absorption spectra of the investigated compounds 1-4 (Tab. I) are the superposition of the corresponding chromophors (Fig. 4b). The partial spectra of the stilbene and the fluorescein (Fig. 4a) or erythrosin are characterized by separated bands. For the selection of 

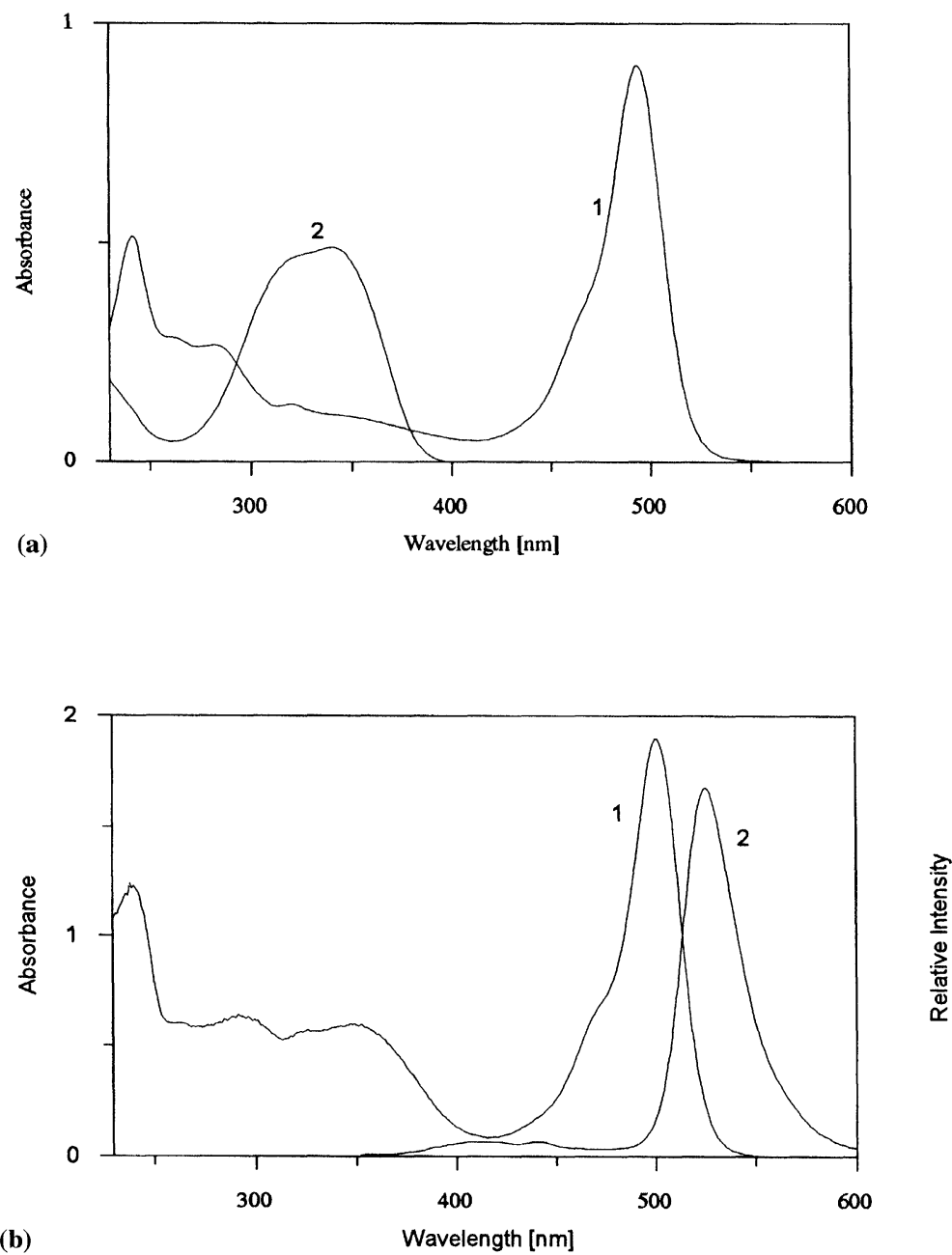

FIGURE 4 Absorption and fluorescence spectra of (a): fluorescein-5-isothiocyanate (1) and 4,4' -diaminostilbene (2); (b): 1; (1)-absorption spectrum; (2)-dual fluorescence spectrum at $\lambda_{\text {exc }}=333 \mathrm{~nm}$.

the model compound 1 and the other investigated $A \sim T \sim A$ systems the following aspects were considered:

1. The energy of the local excited states of $A^{*}$ and $T^{*}$ complies with the precondition shown in Figure 1. 
2. The overlap of the absorption of the antenna chromophor and the fluorescence of the target chromophor (Fig. 4b) is very weak and, therefore, the resonance integral $J$ will be very small (eq. 2). Regardless whether the Förster (eq. 1) or the Dexter (eq. 3) mechanism are considered the rate constant of an excitation energy transfer $T^{*} \rightarrow A$ is very low.

3. Conjugative interaction between the $\pi$-systems of $A$ and $T$ is not transmitted through the thiourea group.

4. The rate constant of the $E, Z$-isomerization is very high, the spectroscopic properties of $E$ - and $Z$-isomer differ [10]. The isomers can be separated using chromatographic methods [11].

\section{Deactivation Behavior at Low Intensity Excitation}

At excitation of 1 using $\lambda_{\mathrm{exc}}=488 \mathrm{~nm}$ (local excitation of the antenna unit) only the fluorescence of a fluorescein derivative is observed (Fig. $4 \mathrm{~b})$. The fluorescence quantum yield is $\varphi_{f}^{488}(\mathbf{1})=0.11 \pm 0.01$. The low quantum yield of 1 compared with fluorescein sodium (Fig. 3) is caused by the heavy atom effect of the covalently bonded sulfur. The intramolecular heavy atom quenching by the sulfur is observed also in the case of other fluorescein thiourea derivatives, in the case of fluorescein-5-isothiocyanate (Fig. 3) and at addition of thiourea to a solution of fluorescein sodium. The Stern-Volmer rate constant of this intermolecular heavy atom quenching is $k_{q}=2.9 \cdot 10^{-9} l \mathrm{~mol}^{-1} \mathrm{~s}^{-1}$.

At excitation of 1 using $\lambda_{\text {exc }}=333 \mathrm{~nm}$ (local excitation of the target unit) a dual fluorescence and a photoreaction are observed simultaneously, in detail:

- the typical fluorescein fluorescence $\varphi_{f, 1}^{333}(\mathbf{1})=0.10 \pm 0.02$,

- a short wavelength fluorescence following to the absorption band of the stilbene chromophore (Fig. 4b), $\varphi_{f, 2}^{333}(\mathbf{1})=0.010 \pm 0.002$,

- the photoisomerization $E-\mathbf{1} \rightarrow Z-\mathbf{1}$ (Fig. 5). $\varphi_{E \rightarrow Z}^{333}(\mathbf{1})=0.020$ \pm 0.004 .

The main deactivation process of the local excited target unit is the undesirable excitation energy transfer $T^{*} \rightarrow A$ (EET). The quantum yields of the corresponding deactivation processes of the isolated excited target chromophor $\left(4,4^{\prime}\right.$-diaminostilbene) are $\varphi_{f}=0.12$ and $\varphi_{E \rightarrow Z}=0.26$. That means about $90 \%$ of the local excited target 


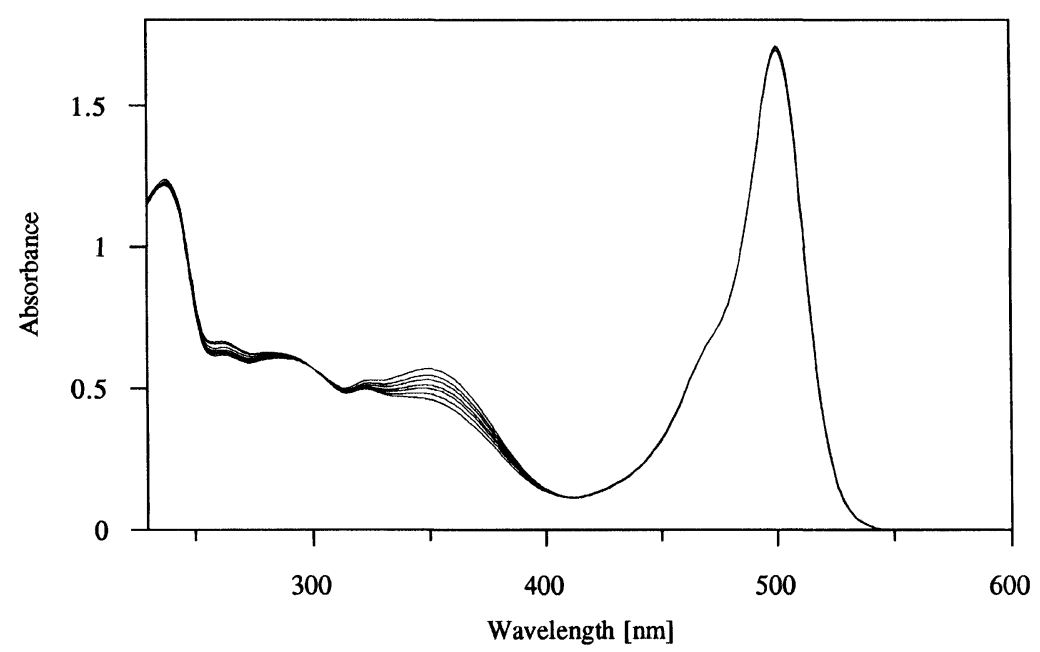

FIGURE 5 Irradiation spectra of $\mathbf{1}$, target excitation at $\lambda_{\mathrm{exc}}=333 \mathrm{~nm}$.

chromophores undergo the excitation energy transfer and only $10 \%$ deactivate via the target specific fluorescence and chemically by photoisomerization. The results of the analysis of the photolyzed solutions using HPLC are shown in Figure 7a. The composition of the photostationary state described by the Zimmerman equation [7] is about $66 \% Z$-isomer and $34 \% E$-isomer.

The compounds 2,3 and $\mathbf{4}$ show a very similar deactivation behavior on excitation at $\lambda_{\mathrm{exc}}=333 \mathrm{~nm}$. In all cases the corresponding dual fluorescence and the isomerization are observed. The fluorescence of the antenna chromophor (fluorescein, erythrosin) is the dominating process.

\section{Deactivation Behavior at High Intensity Excitation}

The photolysis of 1 using the $\mathrm{Ar}^{+}$laser $(488 \mathrm{~nm}, 1.6 \mathrm{~W} / \mathrm{spot} \equiv 14.9 \mathrm{~W}$ $\mathrm{cm}^{-2}$ ) is shown in Figure 6. Beside the very strong fluorescence of the antenna unit the photoisomerization $E-1 \rightarrow Z-1$ goes on. The quantum yield of this photoreaction depends on the intensity of the laser light, the relative quantum yields are

$$
\begin{array}{r}
\varphi_{E \rightarrow Z}^{488}(1600 \mathrm{~mW} / \mathrm{spot}): \varphi_{E \rightarrow Z}^{488}(700 \mathrm{~mW} / \mathrm{spot}): \varphi_{E \rightarrow Z}^{488}(170 \mathrm{~mW} / \mathrm{spot}) \\
=1: 0.2: 0.01 .
\end{array}
$$




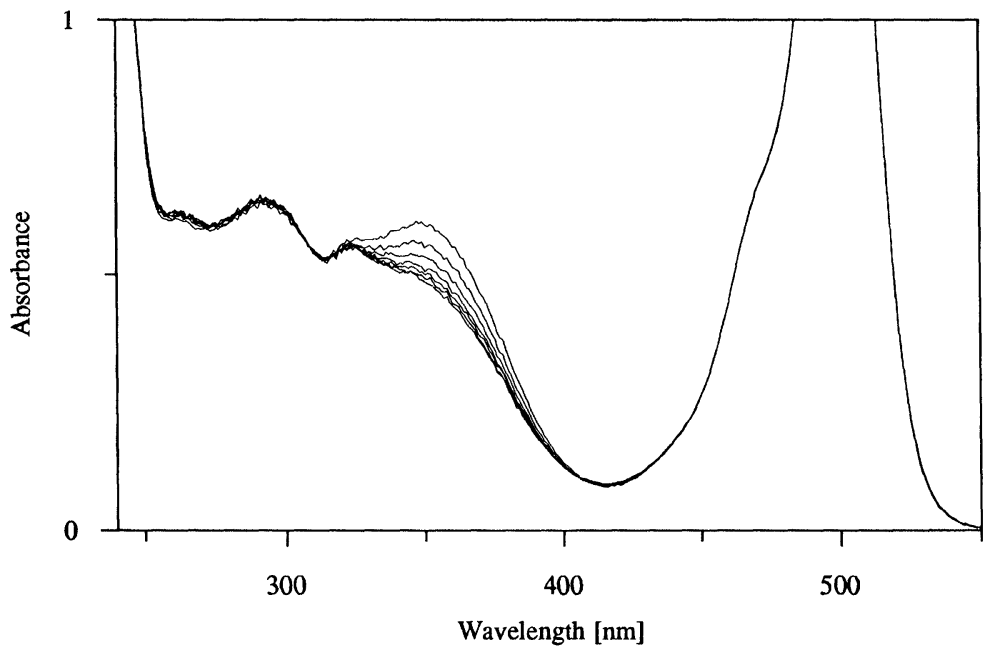

FIGURE 6 Irradiation spectra of 1 , laser excitation of the antenna unit at $\lambda_{\text {exc }}=488 \mathrm{~nm}$ $\left(\mathrm{Ar}^{+}\right.$laser $)$.

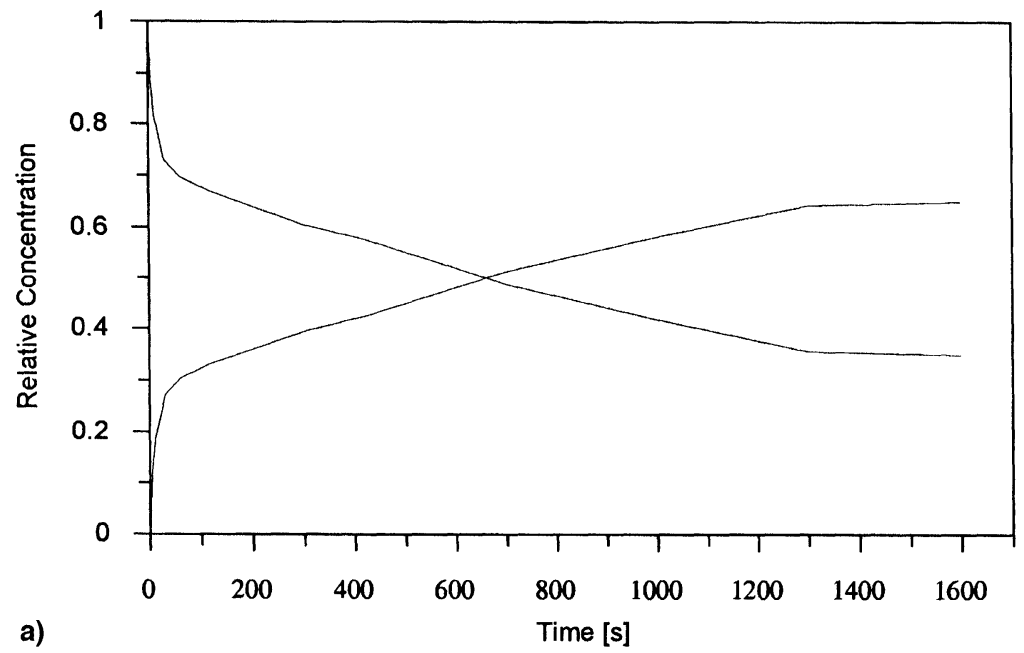

FIGURE 7 Isomerization rate of 1; (a): at lamp excitation (see Fig. 5), (b): at laser excitation (see Fig. 6). 


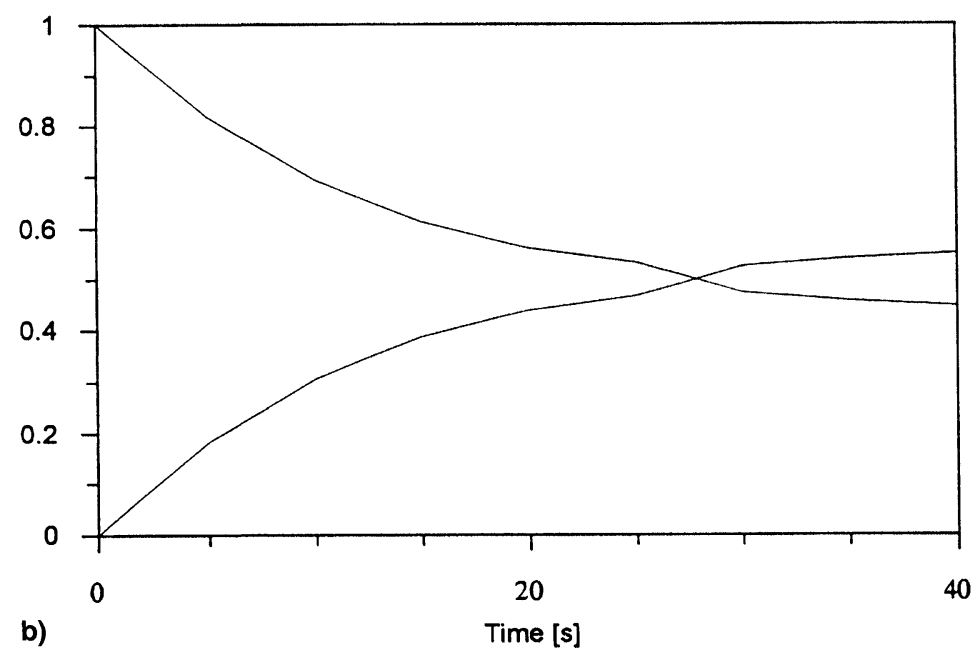

FIGURE 7 (Continued).

The results of the HPLC analysis of the irradiated solutions are presented in Figure $7 \mathrm{~b}$. The composition of the photostationary state at laser photolysis ( $45 \% E$-isomer, $55 \% Z$-isomer) differs from that of lamp photolysis (target excitation, Fig. 7a).

At photolysis of $3\left(\lambda_{\mathrm{exc}}=500 \mathrm{~nm}\right.$, dye laser, or $\lambda_{\mathrm{exc}}=514 \mathrm{~nm}, \mathrm{Ar}^{+}$ laser) the same non-linear behavior of the photoisomerization is observed, but in this case the $A \sim T \sim A$ system is not stable to reach the photostationary state (photobleaching).

The laser irradiation of the reference compounds $\mathbf{2}$ and $\mathbf{4}$ using the same condition demonstrated in Figure 6 does not effect in a photoisomerization. The laserchemical photoisomerization of $\mathbf{1}$ and 3 by antenna excitation is absolutely dependent on the existence of two antenna units.

\section{DISCUSSION}

The scheme 1 illustrates the deactivation processes of the $A \sim T^{*} \sim A$ system populated by monophotonic local excitation of the target (stilbene) unit. The unimolecular processes fluorescence, isomerization and EET compete. 


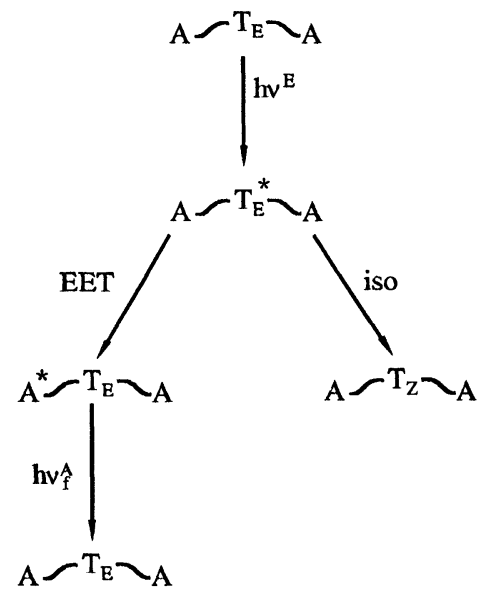

SCHEME 1 Deactivation at target excitation.

The photoisomerization of $\mathbf{1}$ and $\mathbf{3}$ at high intensity antenna excitation is explained by the model of the simultaneous two-photon excitation energy transfer (SEET) shown in scheme 2. The population of the twofold mono-excited state occurs consecutively. For a simultaneous two-photon absorption the used laser intensity is too low. The twofold mono-excited system $A^{*} \sim T \sim A^{*}$ deactivates partly by SEET and populate $A \sim T^{*} \sim A$. The formation of $A \sim T^{*} \sim A$ on this way requires two antenna units, the comparable experiments using 2 and 4 failed, which excludes any mechanism considering a possible two-photon excitation of one chromophor $(A$ or $T)$. The main deactivation process of $A \sim T^{*} \sim A$ is the common EET forming $A \sim T \sim A^{*}$ ("back transfer") followed by the antenna fluorescence.

At the laser irradiation experiment shown in Figure 6. the following experimental conditions were used:

- number of molecules per active volume, determined by concentration, laser spot diameter and thickness of the cuvet: $2.4 \cdot 10^{15}$,

- intensity per spot: $1600 \mathrm{~mW} / \mathrm{spot}$,

- diameter of the spot: $3.70 \mathrm{~mm}$

- photons per second: $9.3 \cdot 10^{17}$.

Assuming a life time of the mono-excited system of about 1 ns (singlet state), during the laser exposure a steady-state concentration of 


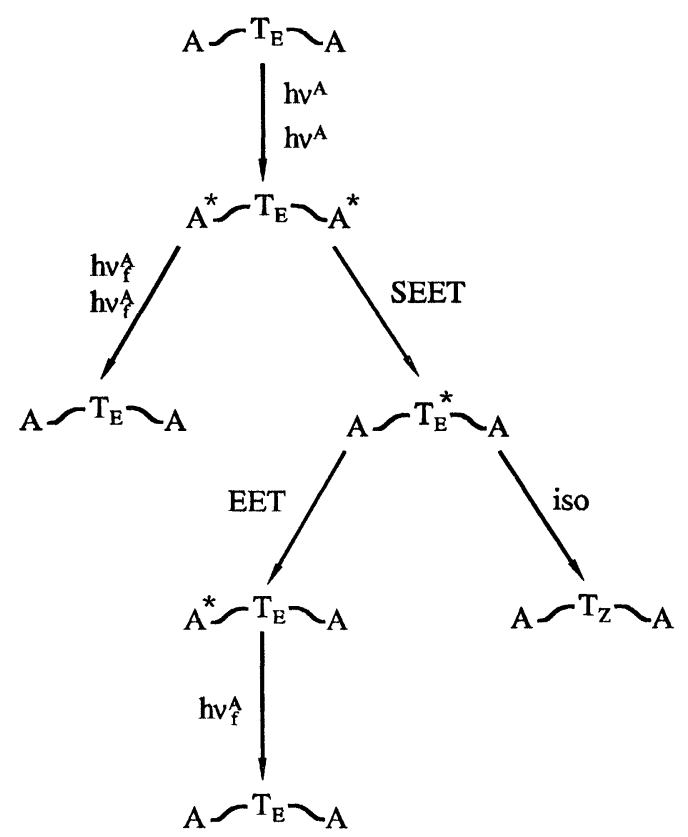

SCHEME 2 Deactivation at high intensity antenna excitation.

about $10^{8}$ molecules per active volume results. This concentration is too low for an effective absorption of a second photon within the life time. From this we conclude, that the absorbing intermediately formed mono-excited states are triplet states. The life time of the fluorescein triplet state $\left(\tau=5 \cdot 10^{-4} \mathrm{~s}\right)$ guarantees a steady-state concentration for $A \sim T \sim A^{*}$ of about $2 \cdot 10^{13}$ molecules per active volume and a second excitation with a probability of 0.0075 . The SEET (from a twofold mono-excited triplet-triplet state) shows similarities to the known triplet-triplet annihilation process.

\section{Acknowledgements}

Financial support by the Deutsche Forschungsgemeinschaft (Bonn), and the Fond der Chemischen Industrie (Frankfurt/Main) is gratefully acknowledged. We thank Dr. J. Stumpe (Humboldt University) for utilization of his laser equipment and M. Rutloh for helpful discussions. 


\section{References}

[1] Bojarski, C. and Sienicki, K. in Photochemistry and Photophysics, Vol. 1 (ed. by J. F. Rabek), CRC press, Inc., Boca Raton, Fa., 1990 p. 1.

[2] Van der Meer, B. W., Coker, G. III and Chen, S.-Y. S. Resonance Energy Transfer, Theory and Data, VCH Publ., Inc., New York 1994.

[3] Förster, Th. (1959). Discuss. Faraday Soc., 27(7).

[4] Dexter, D. L. (1953). J. Chem. Phys., 21, 836.

[5] Haugland, R. P. and Larison, K. D. Handbook of Fluorescent Probes and Research Chemicals, Molecular Probes, Inc., Eugene 1992.

[6] Demas, J. N. and Crosby, C. A. (1971). J. Phys. Chem., 75, 991.

[7] Becker, H. G. O. Einführung in die Photochemie, Deutscher Verlag der Wissenschaften, Berlin 1983.

[8] Becker, W., Dähne, S., Teuchner, K. and Seliger, K. (1975). Exp. Tech. Phys., 23, 297.

[9] Harder, T., Bendig, J., Scholz, G. and Stößer, R. (1996). J. Amer. Chem. Soc., 118, 2497.

[10] Sun, Y. P. and Saltiel, J. (1989). J. Phys. Chem., 93, 8310.

[11] Gauglitz, G., Klink, T. and Schmid, W. (1983). J. Photochem., 22, 285. 